\title{
Generating Quadrilateral and Circular Lattices in KP Theory*
}

\author{
Adam Doliwa $^{1,2}$, Manuel Mañas ${ }^{3,4}$ and Luis Martínez Alonso ${ }^{4}$ \\ ${ }^{1}$ Istituto Nazionale di Fisica Nucleare, Sezione di Roma, \\ P-le. Aldo Moro 2, I-00185, Italy \\ ${ }^{2}$ Instytut Fizyki Teoretycznej, Uniwersytet Warszawski \\ ul. Hoża 69, 00-681 Warszawa, Poland \\ ${ }^{3}$ Departamento de Matemática Aplicada y Estadística, \\ Escuela Universitaria de Ingenieria Técnica Areonaútica, \\ Universidad Politécnica de Madrid, \\ E28040-Madrid, Spain. \\ ${ }^{4}$ Departamento de Física Teórica II, \\ Universidad Complutense, \\ E28040-Madrid, Spain.
}

14th January 2014

\begin{abstract}
The bilinear equations of the $N$-component KP and BKP hierarchies and a corresponding extended Miwa transformation allow us to generate quadrilateral and circular lattices from conjugate and orthogonal nets, respectively. The main geometrical objects are expressed in terms of Baker functions.
\end{abstract}

*Partially supported by CICYT: proyecto PB95-0401 


\section{Introduction}

Among the most interesting developments in Differential Geometry that took place during the last century and the turn of it was the study of orthogonal and conjugate nets [5, 16, 1]. The analysis of orthogonal nets can be already found in Lamé's monograph [25], however it was Darboux who at 1910 wrote a definitive comprehensive text [6] on the subject. Darboux also introduced the notion of conjugate net [5] and studied it in detail. The analysis of the transformations preserving the orthogonal or conjugate character of the net was also developed, for an excellent review see [18]; in this manner, a number of transformations appeared, as for example: Laplace, Lévy 26] and fundamental [22, 17] transformations for conjugate nets, and Ribaucour [35] transformations for orthogonal nets. The iteration of two Ribaucour transformation was performed by Demoulin [10] finding the famous circularity property of these transformations. Recently, the vectorial Ribaucour transformation [28] has been presented and shown to be equivalent to the iteration of the standard ones, see also [19, 20].

Interest from the Soliton Theory groups in this subject is not new; indeed, the deep links among differential geometry and integrable equations has been known for long time. In particular, Zakharov and Manakov [40] were able to devise a $\bar{\partial}$-method for solving the Darboux equations, and later an inverse scattering one for the Lamé equations [39].

Recently, the study of discrete geometry has gained quite an audience. It was in [37] were the two-dimensional quadrilateral lattice was first presented, but it was in [14] were, for the multidimensional case, its integrable character was shown and its transformations were presented [30, 12, 27]. The circular lattices appeared for the first time in [32, 34] and have been studied in detail in a number of papers [2, 1, 12, 24, 11, 29].

In a series of works [13, 31] we have begun studying the relation between these geometrical objects and integrable systems from the point of view of the theory of the multicomponent Kadomtsev-Petviashvili (KP) hierarchy. It is already known that this hierarchy contains a handful of relevant soliton equations like the Davey-Stewartson, the two-dimensional Toda lattice and the $N$-wave resonant interaction system. Among the methods used to describe the KP theory there are two with a remarkable analytical character; i. e., the $\bar{\partial}$ dressing method [40] and the bilinear identity method. The later is closely linked with the infinite Grassmannian manifold [36, 38] and the $\tau$-function fermion formalism [7] (see also [23, 3]). As for our work, we 
find the bilinear approach a most convenient tool for revealing the transformation properties of the KP dynamical objects, like Baker functions or rotation coefficients, under the action of vertex operators. In this way we are able to derive a series of new general identities in the KP theory which constitute the basis of our analysis. As a consequence, we have proved [13] that the multicomponent KP hierarchy can be regarded as the theory of isoconjugate deformations of conjugate nets, and that a similar result [31 for the multicomponent BKP hierarchy and orthogonal nets holds.

In this paper, with the help of some new identities of the KP theory, we show how a standard vertex operator represents a fundamental transformation of the conjugate net. Then, the iteration of this transformation, which leads to a quadrilateral lattice [15], turns out to become a Miwa-like transformation of the KP theory [33]. Such a type of transformations was introduced in [33] to generate the Hirota equation [21] from the KP hierarchy, and then it was extensively used in a series of papers [9] as a method of discretizing soliton equations retaining their integrability. Our analysis provides explicit expressions for the geometrical elements of the generated quadrilateral lattice in terms of Baker functions. Similar results for the multicomponent BKP hierarchy and the circular lattice are also derived by the same means.

The layout of the paper is as follows. In next section we study the fundamental transformation of the conjugate net and introduce the Miwa transformation generating the corresponding quadrilateral lattice, while in $\S 3$ we perform a similar construction of the orthogonal net. In both cases we explicitly give the form of rotation coefficients and tangent vectors of the corresponding lattice in terms of the Baker function.

\section{From conjugate nets to quadrilateral lat- tices}

\subsection{Bilinear formulation of conjugate nets}

The $N$-component KP hierarchy can be formulated in terms of the following bilinear equation involving the $N \times N$ matrix Baker function $\psi$ and its adjoint function $\psi^{*}[7]$

$$
\int_{S^{1}} \psi(z, \boldsymbol{t}) \psi^{*}\left(z, \boldsymbol{t}^{\prime}\right) \mathrm{d} z=0
$$


Here $S^{1}:=\{z \in \mathbb{C}:|z|=1\}$ is the unit circle in the complex plane $\mathbb{C}$, and

$$
\boldsymbol{t}=\left(\boldsymbol{t}_{1}, \ldots, \boldsymbol{t}_{N}\right) \in \mathbb{C}^{N \cdot \infty}, \quad \boldsymbol{t}_{i}:=\left(t_{i, 1}, t_{i, 2}, \ldots\right) \in \mathbb{C}^{\infty} .
$$

It is also assumed that $\psi$ and $\psi^{*}$ can be factorized as

$$
\begin{aligned}
\psi(z, \boldsymbol{t}) & :=\chi(z, \boldsymbol{t}) \psi_{0}(z, \boldsymbol{t}), \\
\psi^{*}(z, \boldsymbol{t}) & :=\psi_{0}(z, \boldsymbol{t})^{-1} \chi^{*}(z, \boldsymbol{t}),
\end{aligned}
$$

where the bare Baker functions $\chi$ and $\chi^{*}$ admit Laurent expansions on $S^{1}$, the first terms of which being

$$
\begin{aligned}
\chi(z) & =1+\beta z^{-1}+\mathcal{O}\left(z^{-2}\right), \\
\chi^{*}(z) & =1-\beta z^{-1}+\mathcal{O}\left(z^{-2}\right) .
\end{aligned}
$$

Notice that these Laurent series can be analitically extended from $S^{1}$ to the region $|z|>1$. The vacuum Baker function $\psi_{0}$ is defined as

$$
\psi_{0}(z, \boldsymbol{t})=\exp \left(\sum_{i=1}^{N} \xi\left(z, \boldsymbol{t}_{i}\right) P_{i}\right), \quad \xi\left(z, \boldsymbol{t}_{i}\right):=\sum_{n=1}^{\infty} z^{n} t_{i, n},
$$

with $P_{i}:=\left(\delta_{i k} \delta_{i l}\right)_{k, l=1, \ldots, N}$ being the projection matrix in the $i$-th direction.

The identity (1) encodes the Grassmannian formulation of the KP hierarchy [36, 38] and it leads at once to the KP linear system of equations for $\psi$ and $\psi^{*}$. Let us, for instance, outline this approach for the Baker function $\psi$. To this end, we denote by $W$ the set of $N \times N$ matrix functions $\varphi(z)$ such that:

$$
\int_{S^{1}} \varphi(z) \psi^{*}\left(z, \boldsymbol{t}^{\prime}\right) \mathrm{d} z=0,
$$

for all $\boldsymbol{t}^{\prime}$ in the definition domain of $\psi^{*}$. Under appropriate conditions $W$ belongs to an infinite-dimensional Grassmannian manifold [36, 38]. From (11) it follows that $W$ is a left $M_{N}(\mathbb{C})$-module, with $M_{N}(\mathbb{C})$ being the ring of $N \times N$ complex matrices. Moreover, as a consequence of ([1) and (3) one has that for any $\boldsymbol{t}$ :

$$
W=\bigoplus_{n \geq 0} M_{N}(\mathbb{C}) \cdot v_{n}(\boldsymbol{t}), \quad v_{n}(z, \boldsymbol{t})=\left(\sum_{k=1}^{N} \frac{\partial}{\partial u_{k}}\right)^{n} \psi(z, \boldsymbol{t}),
$$


where $u_{k}:=t_{k, 1}, k=1, \ldots, N$; notice also that on $S^{1}$ we have

$$
v_{n}(z)=\left(z^{n}+\mathcal{O}\left(z^{n-1}\right)\right) \psi_{0}(z) .
$$

The linear system for $\psi$ results from the decompositions of the time derivatives of $\psi$ in terms of $v_{n}, n=0, \ldots, \infty$. In particular, from $P_{i} \partial \psi / \partial u_{k}$, $i \neq k$, one gets

$$
\frac{\partial \psi_{i j}}{\partial u_{k}}=\beta_{i k} \psi_{k j}
$$

In a similar way it follows that the adjoint Baker function satisfies

$$
\frac{\partial \psi_{i j}^{*}}{\partial u_{k}}=\beta_{k j} \psi_{i k}^{*} .
$$

The compatibility of either (6) or (7) leads to the Darboux equations for the rotation coefficients $\beta_{i j}, i, j=1, \ldots, N$ :

$$
\frac{\partial \beta_{i j}}{\partial u_{k}}=\beta_{i k} \beta_{k j}, \quad k \neq i, j .
$$

One may recognize here the tangent vectors $\boldsymbol{X}_{i}$ of the conjugate net which can be expressed as

$$
\boldsymbol{X}_{i}=\int_{S^{1}} \boldsymbol{\psi}_{i}(z) f(z) \mathrm{d} z
$$

where $\boldsymbol{\psi}_{i}$ denotes the $i$-th row of $\psi$ and $f=\operatorname{diag}\left(f_{1}, \ldots, f_{N}\right)$ is a suitable diagonal distribution matrix. In a similar way, the Lamé coefficients can be constructed as follows

$$
H_{i}(\boldsymbol{t})=\sum_{j=1}^{N} \int_{S^{1}} h_{j}(z) \psi_{j i}^{*}(z, \boldsymbol{t}) \mathrm{d} z, \quad i=1, \cdots, N,
$$

for suitable distributions $\left\{h_{j}\right\}_{j=1, \ldots, N}$. Obviously the higher times $\boldsymbol{t}_{2}, \boldsymbol{t}_{3}, \ldots$ can be considered as iso-conjugate deformation parameters [13].

An essential element in the subsequent discussion is the vertex operator

$$
\mathbb{V}^{(i)}(p, q) f(\boldsymbol{t}):=f\left(\boldsymbol{t}+([p]-[q]) \boldsymbol{e}_{i}\right)
$$

where $\left\{\boldsymbol{e}_{i}\right\}_{i=1}^{N}$ is the canonical basis of $\mathbb{C}^{N}$ and

$$
[z]:=\left(\frac{1}{z}, \frac{1}{2 z^{2}}, \frac{1}{3 z^{3}}, \ldots\right) .
$$

As it is proved below, from a geometrical point of view this operator represents a fundamental transformation of the conjugate net. 


\subsection{Action of vertex operators on Baker functions}

The bilinear identity (11) is a useful tool for obtaining the transformation properties of the rotation coefficients and the Baker functions under the action of vertex operators. We first observe that given $p, q \in \mathbb{C}$ such that $|p|,|q|>1$, then for all $z \in S^{1}$ it follows that

$$
\mathbb{V}^{(i)}(p, q) \psi(z, \boldsymbol{t})=\left[\mathbb{V}^{(i)}(p, q) \chi(z, \boldsymbol{t})\right] \psi_{0}(z, \boldsymbol{t})\left[1-\left(1-\frac{p}{q}\right) \frac{z}{z-p} P_{i}\right]
$$

so that $\mathbb{V}^{(i)}(p, q) \psi(z)$ can be meromorphically extended to the region with $|z| \geq 1$ being the unique singularity a simple pole located at $z=p$. The corresponding residue satisfies

$$
\operatorname{Res}_{p}\left[\mathbb{V}^{(i)}(p, q) \psi\right]\left(1-P_{i}\right)=0
$$

In this way, if we perform the substitutions $\boldsymbol{t} \rightarrow \boldsymbol{t}+([p]-[q]) \boldsymbol{e}_{i}$ and $\boldsymbol{t}^{\prime} \boldsymbol{\rightarrow} \boldsymbol{t}$ in (1) and evaluate the resulting integral, the only non-vanishing contributions come from residues at $z=\infty$ and $z=p$. Hence we find

$$
\begin{array}{r}
-p\left(1-\frac{p}{q}\right) P_{i}+\left[\mathbb{V}^{(i)}(p, q) \beta\right]\left[1-\left(1-\frac{p}{q}\right) P_{i}\right]-\left[1-\left(1-\frac{p}{q}\right) P_{i}\right] \beta \\
=\operatorname{Res}_{p}\left[\mathbb{V}^{(i)}(p, q) \psi\right] \psi^{*}(p)
\end{array}
$$

In the following diagram we show the polar structure and the contour of integration in the Riemann sphere

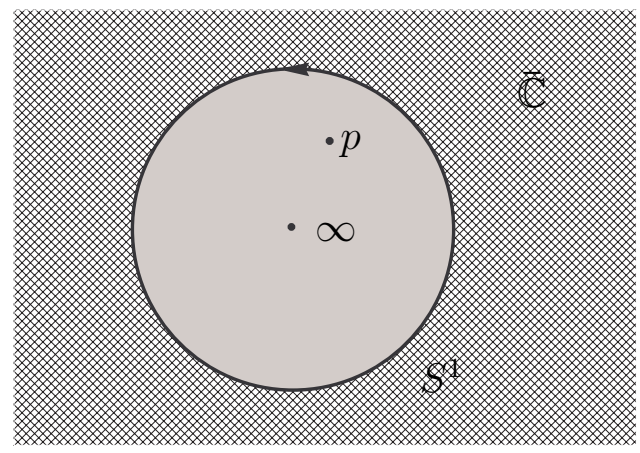

The components of this matrix equation can be obtained by multiplying (13) to the left and to the right by the projectors $P_{j}, i=1, \ldots, N$. Thus, 
one finds the following identities for the action of $\mathbb{V}^{(i)}(p, q)$ on the rotation coefficients

$$
\begin{aligned}
\mathbb{V}^{(i)}(p, q) \beta_{i i} & =\beta_{i i}+q\left(1-\frac{p}{q}\right)+\frac{q}{p} \operatorname{Res}_{p}\left(\mathbb{V}^{(i)}(p, q) \psi_{i i}\right) \psi_{i i}^{*}(p), \\
\frac{q}{p} \mathbb{V}^{(i)}(p, q) \beta_{i j} & =\beta_{i j}+\frac{q}{p} \operatorname{Res}_{p}\left(\mathbb{V}^{(i)}(p, q) \psi_{i i}\right) \psi_{i j}^{*}(p), \quad i \neq j, \\
\frac{p}{q} \mathbb{V}^{(i)}(p, q) \beta_{j i} & =\beta_{j i}+\operatorname{Res}_{p}\left(\mathbb{V}^{(i)}(p, q) \psi_{j i}\right) \psi_{i i}^{*}(p), \quad i \neq j, \\
\mathbb{V}^{(i)}(p, q) \beta_{j k} & =\beta_{j k}+\operatorname{Res}_{p}\left(\mathbb{V}^{(i)}(p, q) \psi_{j i}\right) \psi_{i k}^{*}(p), \quad i, j \text { and } k \text { different. }
\end{aligned}
$$

Our next aim is to derive similar identities for the Baker function $\psi$. To this end we consider the function

$$
F:=P_{j}\left[\frac{\operatorname{Res}_{p}\left(\mathbb{V}^{(i)}(p, q) \psi\right)}{\operatorname{Res}_{p}\left(\mathbb{V}^{(i)}(p, q) \psi_{i i}\right)}-1\right] \mathbb{V}^{(i)}(p, q) \psi,
$$

and observe that its unique possible singularity in the region $|z|>1$ might be located at $z=p$. However, it is easy to see that (12) implies that $z=p$ is a removable singularity of $F$. Therefore, the function $F$ can be analytically extended from $S^{1}$ to the region $|z|>1$. Furthermore, from (11) it follows that

$$
\mathbb{V}^{(i)}(p, q) \psi(z) \sim\left(1-\left(1-\frac{p}{q}\right) P_{i}+\mathcal{O}\left(z^{-1}\right)\right) \psi_{0}(z), \quad z \rightarrow \infty
$$

so that the first terms of the Laurent expansion of $F$ on $S^{1}$ are

$$
F=P_{j}\left[\frac{\operatorname{Res}_{p}\left(\mathbb{V}^{(i)}(p, q) \psi\right)}{\operatorname{Res}_{p}\left(\mathbb{V}^{(i)}(p, q) \psi_{i i}\right)}-1\right]\left(1-\left(1-\frac{p}{q}\right) P_{i}+\mathcal{O}\left(z^{-1}\right)\right) \psi_{0}(z) .
$$

Hence from the uniqueness of the Baker function in the Grassmannian element $W$ we deduce

$P_{j}\left[\frac{\operatorname{Res}_{p}\left(\mathbb{V}^{(i)}(p, q) \psi\right)}{\operatorname{Res}_{p}\left(\mathbb{V}^{(i)}(p, q) \psi_{i i}\right)}-1\right] \mathbb{V}^{(i)}(p, q) \psi(z)=P_{j}\left[\frac{p}{q} \frac{\operatorname{Res}_{p}\left(\mathbb{V}^{(i)}(p, q) \psi\right)}{\operatorname{Res}_{p}\left(\mathbb{V}^{(i)}(p, q) \psi_{i i}\right)}-1\right] \psi(z)$

In terms of the rows $\boldsymbol{\psi}_{j}$, this identity can be rewritten as

$$
\mathbb{V}^{(i)}(p, q) \boldsymbol{\psi}_{j}=\boldsymbol{\psi}_{j}+\frac{\operatorname{Res}_{p}\left(\mathbb{V}^{(i)}(p, q) \psi_{j i}\right)}{\operatorname{Res}_{p}\left(\mathbb{V}^{(i)}(p, q) \psi_{i i}\right)}\left(\mathbb{V}^{(i)}(p, q) \boldsymbol{\psi}_{i}-\frac{p}{q} \boldsymbol{\psi}_{i}\right), \quad j \neq i .
$$

By noticing now that (11) implies that $\mathbb{V}^{(i)}(p, q) \psi(q) P_{i}=0$, we get from (19) the following useful identity

$$
\frac{\psi_{j i}(q)}{\operatorname{Res}_{p}\left(\mathbb{V}^{(i)}(p, q) \psi_{j i}\right)}=\frac{p}{q} \frac{\psi_{i i}(q)}{\operatorname{Res}_{p}\left(\mathbb{V}^{(i)}(p, q) \psi_{i i}\right)}, \quad j \neq i
$$




\subsection{From vertex operators to fundamental transforma- tions}

With the help of the above identities it is now straightforward to formulate a representation of a fundamental transformation as a vertex operator. We first prove that the following operator acting on the rotation coefficients

$$
\begin{aligned}
\mathcal{F}^{(i)}\left(\beta_{i j}\right) & :=\frac{q}{p} \mathbb{V}^{(i)}(p, q) \beta_{i j}, \quad i \neq j \\
\mathcal{F}^{(i)}\left(\beta_{j i}\right) & :=\frac{p}{q} \mathbb{V}^{(i)}(p, q) \beta_{j i}, \quad i \neq j \\
\mathcal{F}^{(i)}\left(\beta_{j k}\right) & :=\mathbb{V}^{(i)}(p, q) \beta_{j k}, \quad i, j \text { and } k \text { different } .
\end{aligned}
$$

is a fundamental transformation.

In view of (14 17 ) and taking $(20)$ into account, the action of $\mathcal{F}^{(i)}$ can be expressed as

$$
\mathcal{F}^{(i)}\left(\beta_{j k}\right)=\beta_{j k}-\frac{v_{j}^{(i)} v_{k}^{(i)^{*}}}{\Omega^{(i)}},
$$

where

$$
\begin{aligned}
v_{j}^{(i)} & :=\psi_{j i}(q) \\
v_{k}^{(i)^{*}} & :=\psi_{i k}^{*}(p), \\
\Omega^{(i)} & :=-\frac{p}{q} \frac{\psi_{i i}(q)}{\operatorname{Res}_{p}\left(\mathbb{V}^{(i)}(p, q) \psi_{i i}\right)}
\end{aligned}
$$

Obviously, $v_{j}^{(i)}$ and $v_{k}^{(i)^{*}}$ are solutions of the linear systems (6) and (7). Therefore, in order to conclude that $\mathcal{F}^{(i)}$ is a fundamental transformation with data given by (21) we have to show that $\Omega^{(i)}$ is a potential, i. e.:

$$
\frac{\partial \Omega^{(i)}}{\partial u_{j}}=v_{j}^{(i)} v_{j}^{(i)^{*}} .
$$

For example, for $j \neq i$, the use of (6), (20) and (15) leads to the desired result. The $i$-th derivative must be treated with more care, in fact, we have to start with the alternative expressions

$$
\Omega^{(i)}=\frac{\psi_{j i}(q)}{\operatorname{Res}_{p}\left(\mathbb{V}^{(i)}(p, q) \psi_{j i}\right)}, \quad j \neq i ;
$$


take the $i$-th derivative and use (16).

As for the fundamental transformation acting on the tangent vectors of the net, it is given by

$$
\begin{aligned}
\mathcal{F}^{(i)}\left(\boldsymbol{X}_{i}\right) & :=\frac{q}{p} \mathbb{V}^{(i)}(p, q) \boldsymbol{X}_{i}, \\
\mathcal{F}^{(i)}\left(\boldsymbol{X}_{j}\right) & :=\mathbb{V}^{(i)}(p, q) \boldsymbol{X}_{j}, \quad j \neq i,
\end{aligned}
$$

which can be written as

$$
\mathcal{F}^{(i)}\left(\boldsymbol{X}_{j}\right)=\boldsymbol{X}_{j}-\frac{v_{j}^{(i)}}{\Omega^{(i)}} \mathfrak{e}^{(i)},
$$

where

$$
\mathcal{C}^{(i)}:=\frac{\mathbb{V}^{(i)}(p, q) \boldsymbol{X}_{i}-\frac{p}{q} \boldsymbol{X}_{i}}{\operatorname{Res}_{p}\left(\mathbb{V}^{(i)}(p, q) \psi_{i i}\right)},
$$

is clearly the Combescure vector of this fundamental geometrical transformation: in fact a simple check shows that

$$
\frac{\partial \mathfrak{C}^{(i)}}{\partial u_{j}}=\boldsymbol{X}_{j} v_{j}^{(i)^{*}} .
$$

For $j \neq i$ one just take the derivative and then uses (9), (6), (20) and (15). The $j=i$ case requires the use of the following expression for $\mathbf{e}^{(i)}$ (it derives from (201))

$$
\mathfrak{C}^{(i)}=\frac{\mathbb{V}^{(i)}(p, q) \boldsymbol{X}_{j}-\boldsymbol{X}_{j}}{\operatorname{Res}_{p}\left(\mathbb{V}^{(i)}(p, q) \psi_{j i}\right)}
$$

and formulae (9), (6) and (16).

\subsection{Miwa transformations and the quadrilateral lattice}

We are going to see how the quadrilateral lattice is generated from the conjugate net by discretizing variables in the Baker functions by means of a Miwa like transformation [27]

$$
\begin{aligned}
\Psi(z, \boldsymbol{t}, \boldsymbol{n}) & :=\psi(z, \boldsymbol{t}+\boldsymbol{n}([p]-[q])), \\
\Psi^{*}(z, \boldsymbol{t}, \boldsymbol{n}) & :=\psi^{*}(z, \boldsymbol{t}+\boldsymbol{n}([p]-[q])),
\end{aligned}
$$


where $\boldsymbol{n}=n_{1} \boldsymbol{e}_{1}+\cdots+n_{N} \boldsymbol{e}_{N} \in \mathbb{Z}^{N}$. Notice that from the point of view of our above discussion the discrete variables in (22) are introduced by a composition of fundamental transformations acting on tangent vectors of the conjugate net. Therefore, it is known [15] that as a consequence the corresponding lattice is a quadrilateral lattice with suitable re-normalized tangent vectors given by the Combescure vectors $\left\{\mathbf{C}^{(i)}\right\}_{i=1}^{N}$. However, finding the quadrilateral rotation coefficients $Q_{i j}$ requires to consider the iteration $\mathcal{F}^{(i)} \circ \mathcal{F}^{(j)}=: \mathcal{F}^{(i, j)}$. For that aim we consider the composed vertex operator $\mathbb{V}^{(i, j)}(p, q)=\mathbb{V}^{(i)}(p, q) \circ \mathbb{V}^{(j)}(p, q)$.

Since

$$
\mathbb{V}^{(i, j)}(p, q) \psi(z, \boldsymbol{t})=\left[\mathbb{V}^{(i, j)}(p, q) \chi(z, \boldsymbol{t})\right] \psi_{0}(z, \boldsymbol{t})\left[1-\left(1-\frac{p}{q}\right) \frac{z}{z-p}\left(P_{i}+P_{j}\right)\right]
$$

we have a simple pole appearing at $z=p$. In fact this equation is (11) after the replacement of the projector $P_{i}$ by the projector $P_{i}+P_{j}$. Thus by substituting $\boldsymbol{t} \rightarrow \boldsymbol{t}+([p]-[q])\left(\boldsymbol{e}_{i}+\boldsymbol{e}_{j}\right)$ and $\boldsymbol{t}^{\prime} \rightarrow \boldsymbol{t}$ in (11), the only non-vanishing contributions to the integral come from $z=\infty$ and $z=p$,so

$$
\begin{gathered}
-p\left(1-\frac{p}{q}\right)\left(P_{i}+P_{j}\right)+\left[\mathbb{V}^{(i, j)}(p, q) \beta\right]\left[1-\left(1-\frac{p}{q}\right)\left(P_{i}+P_{j}\right)\right]-\left[1-\left(1-\frac{p}{q}\right)\left(P_{i}+P_{j}\right)\right] \beta \\
=\operatorname{Res}_{p}\left[\mathbb{V}^{(i, j)}(p, q) \psi\right] \psi^{*}(p) .
\end{gathered}
$$

We introduce now some convenient notation

$$
\begin{gathered}
B:=\left(\begin{array}{ll}
\beta_{i i} & \beta_{i j} \\
\beta_{j i} & \beta_{j j}
\end{array}\right), \quad b_{k}:=\left(\begin{array}{c}
\beta_{i k} \\
\beta_{j k}
\end{array}\right), \quad \tilde{b}_{k}:=\left(\begin{array}{ll}
\beta_{k i} & \beta_{k j}
\end{array}\right), \\
\Phi:=\left(\begin{array}{cc}
\psi_{i i} & \psi_{i j} \\
\psi_{j i} & \psi_{j j}
\end{array}\right), \quad \phi_{k}:=\left(\begin{array}{c}
\psi_{i k} \\
\psi_{j k}
\end{array}\right), \quad \tilde{\phi}_{k}:=\left(\begin{array}{ll}
\psi_{k i} & \psi_{k j}
\end{array}\right),
\end{gathered}
$$

which allows us to express the components of the matrix equation (24) as

$$
\begin{aligned}
\mathbb{V}^{(i, j)}(p, q) B & =B+q\left(1-\frac{p}{q}\right)+\frac{q}{p} \operatorname{Res}_{p}\left(\mathbb{V}^{(i, j)}(p, q) \Phi\right) \Phi^{*}(p), \\
\frac{q}{p} \mathbb{V}^{(i, j)}(p, q) b_{k} & =b_{k}+\frac{q}{p} \operatorname{Res}_{p}\left(\mathbb{V}^{(i, j)}(p, q) \Phi\right) \phi_{k}^{*}(p), \quad i, j \text { and } k \text { different, } \\
\frac{p}{q} \mathbb{V}^{(i, j)}(p, q) \tilde{b}_{k} & =\tilde{b}_{k}+\operatorname{Res}_{p}\left(\mathbb{V}^{(i, j)}(p, q) \tilde{\phi}_{k}\right) \Phi^{*}(p), \quad i, j \text { and } k \text { different, } \\
\mathbb{V}^{(i, j)}(p, q) \beta_{k l} & =\beta_{k l}+\operatorname{Res}_{p}\left(\mathbb{V}^{(i, j)}(p, q) \tilde{\phi}_{k}\right) \phi_{k}^{*}(p), \quad i, j, k \text { and } l \text { different. }
\end{aligned}
$$


We aim now to get similar identities for the Baker function. From (23) it is clear that

$$
\operatorname{Res}_{p}\left(\mathbb{V}^{(i, j)}(p, q) \psi\right)=\operatorname{Res}_{p}\left(\mathbb{V}^{(i, j)}(p, q) \psi\right)\left(P_{i}+P_{j}\right)
$$

that implies

$$
\operatorname{Res}_{p}\left(P_{k}\left[\operatorname{Res}_{p}\left(\mathbb{V}^{(i, j)}(p, q) \psi\right) \rho_{i j}\left[\left(\operatorname{Res}_{p}\left(\mathbb{V}^{(i, j)}(p, q) \Phi\right)\right)^{-1}\right]-1\right] \mathbb{V}^{(i, j)}(p, q) \psi\right)=0
$$

where $\rho_{i j}: M_{2 \times 2} \rightarrow M_{N \times N}$ with $\rho_{i j}\left[\left(m_{a b}\right)_{a, b=i, j}\right]=\left(m_{a b} \delta_{a k} \delta_{b l}\right)_{\substack{k, l=1, \ldots, N \\ a, b=i, j}}$ is the canonical embedding of $2 \times 2$ matrices into $N \times N$ matrices in the cross of the $i$-th and $j$-th columns and rows.

The same arguments as in the previous subsection lead us to the identity

$$
\begin{aligned}
P_{k}\left[\operatorname{Res}_{p}\left(\mathbb{V}^{(i, j)}(p, q) \psi\right) \rho_{i j}\left[\left(\operatorname{Res}_{p}\left(\mathbb{V}^{(i, j)}(p, q) \Phi\right)\right)^{-1}\right]-1\right] \mathbb{V}^{(i, j)}(p, q) \psi \\
\quad=P_{k}\left[\frac{p}{q} \operatorname{Res}_{p}\left(\mathbb{V}^{(i, j)}(p, q) \psi\right) \rho_{i j}\left[\left(\operatorname{Res}_{p}\left(\mathbb{V}^{(i, j)}(p, q) \Phi\right)^{-1}\right]-1\right] \psi,\right.
\end{aligned}
$$

which in terms of the rows of $\psi$ reads

$$
\begin{aligned}
& \mathbb{V}^{(i, j)}(p, q) \boldsymbol{\psi}_{k}=\boldsymbol{\psi}_{k}+\operatorname{Res}_{p}\left(\mathbb{V}^{(i, j)}(p, q) \tilde{\phi}_{k}\right) \\
& \times\left[\operatorname{Res}_{p}\left(\mathbb{V}^{(i, j)}(p, q) \Phi\right)\right]^{-1}\left(\begin{array}{c}
\mathbb{V}^{(i, j)}(p, q) \boldsymbol{\psi}_{i}-\frac{p}{q} \boldsymbol{\psi}_{i} \\
\mathbb{V}^{(i, j)}(p, q) \boldsymbol{\psi}_{j}-\frac{p}{q} \boldsymbol{\psi}_{j}
\end{array}\right) .
\end{aligned}
$$

Finally, from (23) it follows that $\mathbb{V}^{(i, j)}(p, q) \psi(q)\left(P_{i}+P_{j}\right)=0$, that together with (31) gives

$$
\tilde{\phi}_{k}(q) \Phi(q)^{-1}=\frac{p}{q} \operatorname{Res}_{p}\left(\mathbb{V}^{(i, j)}(p, q) \tilde{\phi}_{k}\right)\left[\operatorname{Res}_{p}\left(\mathbb{V}^{(i, j)}(p, q) \Phi\right)\right]^{-1}, \quad k \neq i, j .
$$

Identification with the iterated fundamental transformation As it is known [15] the vectorial fundamental transformation is just a composition of two different fundamental transformations. It turns out that the following operator acting on the rotation coefficients

$$
\begin{aligned}
\mathcal{F}^{(i, j)}\left(b_{k}\right) & :=\frac{q}{p} \mathbb{V}^{(i, j)}(p, q) b_{k}, \quad k \neq i, j, \\
\mathcal{F}^{(i, j)}\left(\tilde{b}_{k}\right) & :=\frac{p}{q} \mathbb{V}^{(i, j)}(p, q) \tilde{b}_{k}, \quad k \neq i, j, \\
\mathcal{F}^{(i, j)}\left(\beta_{k l}\right) & :=\mathbb{V}^{(i, j)}(p, q) \beta_{k, l}, \quad i, j, k \text { and } l \text { different, }
\end{aligned}
$$


is a iterated (or vectorial) fundamental transformation. Indeed, from (25-28) we can write

$$
\mathcal{F}^{(i, j)}\left(\beta_{k l}\right)=\beta_{k l}-\tilde{\phi}_{k}(q) \Omega^{(i, j)^{-1}} \phi_{l}^{*}(p),
$$

where

$$
\Omega^{(i, j)}:=-\frac{p}{q}\left[\operatorname{Res}_{p}\left(\mathbb{V}^{(i, j)}(p, q) \Phi\right)\right]^{-1} \Phi(q)
$$

As in the previous section is not difficult to check that $\Omega^{(i, j)}$ is a potential matrix; i. e.,

$$
\frac{\partial \Omega^{(i, j)}}{\partial u_{k}}=\phi_{k}^{*}(p) \tilde{\phi}_{k}(q)
$$

As for the vectorial fundamental transformation of the tangent vectors, it takes the form

$$
\begin{aligned}
& \mathcal{F}^{(i, j)}\left(\boldsymbol{X}_{k}\right):=\frac{q}{p} \mathbb{V}^{(i, j)}(p, q) \boldsymbol{X}_{k}, \quad k=i, j, \\
& \mathcal{F}^{(i, j)}\left(\boldsymbol{X}_{k}\right):=\mathbb{V}^{(i, j)}(p, q) \boldsymbol{X}_{k}, \quad k \neq i, j,
\end{aligned}
$$

Observe that according to (31) we have

$$
\mathcal{F}^{(i, j)}\left(\boldsymbol{X}_{k}\right)=\boldsymbol{X}_{k}-\tilde{\phi}_{k} \Omega^{(i, j)^{-1}}\left[\operatorname{Res}_{p}\left(\mathbb{V}^{(i, j)}(p, q) \Phi\right)\right]^{-1}\left(\begin{array}{c}
\mathbb{V}^{(i, j)}(p, q) \boldsymbol{X}_{i}-\frac{p}{q} \boldsymbol{X}_{i} \\
\mathbb{V}^{(i, j)}(p, q) \boldsymbol{X}_{j}-\frac{p}{q} \boldsymbol{X}_{j}
\end{array}\right)
$$

Now, we observe that

$$
\rho_{i j}\left[\left(\operatorname{Res}_{p}\left(\mathbb{V}^{(i, j)}(p, q) \Phi\right)\right)^{-1}\right] \mathbb{V}^{(i, j)}(p, q) \psi-\sum_{k=i, j} P_{k} \frac{\mathbb{V}^{(k)}(p, q) \psi}{\operatorname{Res}_{p}\left(\mathbb{V}^{(k)}(p, q) \psi_{k k}\right)}
$$

is an analytical function in the region $|z| \geq 1$ up to a possible simple pole at $z=p$; however, we see that its residue there vanishes and therefore $z=p$ is a removable singularity. Thus, the above function is holomorphic outside the unit circle. Taking into account its asymptotic expansion at $z=\infty$ and 
the uniqueness of the Baker function in the corresponding element in the Grassmannian we deduce:

$$
\begin{aligned}
\rho_{i j}\left[\left(\operatorname{Res}_{p}\left(\mathbb{V}^{(i, j)}(p, q) \Phi\right)\right)^{-1}\right] \mathbb{V}^{(i, j)}(p, q) \psi-\sum_{k=i, j} P_{k} \frac{\mathbb{V}^{(k)}(p, q) \psi}{\operatorname{Res}_{p}\left(\mathbb{V}^{(k)}(p, q) \psi_{k k}\right)} \\
=\frac{p}{q}\left(\rho_{i j}\left[\left(\operatorname{Res}_{p}\left(\mathbb{V}^{(i, j)}(p, q) \Phi\right)\right)^{-1}\right]-\sum_{k=i, j} \frac{P_{k}}{\operatorname{Res}_{p}\left(\mathbb{V}^{(k)}(p, q) \psi_{k k}\right)}\right) \psi
\end{aligned}
$$

From the rows of this matrix relation we obtain

$$
\left[\operatorname{Res}_{p}\left(\mathbb{V}^{(i, j)}(p, q) \Phi\right)\right]^{-1}\left(\begin{array}{c}
\mathbb{V}^{(i, j)}(p, q) \boldsymbol{X}_{i}-\frac{p}{q} \boldsymbol{X}_{i} \\
\mathbb{V}^{(i, j)}(p, q) \boldsymbol{X}_{j}-\frac{p}{q} \boldsymbol{X}_{j}
\end{array}\right)=\left(\begin{array}{l}
\boldsymbol{e}^{(i)} \\
\boldsymbol{e}^{(j)}
\end{array}\right)
$$

and we have

$$
\mathcal{F}^{(i, j)}\left(\boldsymbol{X}_{k}\right)=\boldsymbol{X}_{k}-\tilde{\phi}_{k} \Omega^{(i, j)^{-1}}\left(\begin{array}{l}
\boldsymbol{C}^{(i)} \\
\boldsymbol{C}^{(j)}
\end{array}\right)
$$

The above formula is just the vectorial fundamental transformation for the tangent vectors of the conjugate net.

Now, since the fundamental transformation of the Combescure vectors satisfies

$$
\mathcal{F}^{(j)}\left(\boldsymbol{C}^{(i)}\right)=\boldsymbol{C}^{(i)}-\frac{\Omega_{i j}^{(i, j)}}{\Omega_{j j}^{(i, j)}} \boldsymbol{C}^{(j)}, \quad i \neq j
$$

then by performing the discretization (22) the transformation $\mathcal{F}^{(j)}$ becomes the shift $T_{j}$ in the $n_{j}$ discrete variable. In this way we obtain the desired quadrilateral lattice equations

$$
\Delta_{j} \mathfrak{e}^{(i)}=\left(T_{j} Q_{i j}\right) \mathfrak{C}^{(j)}, \quad i \neq j,
$$

with

$$
T_{j} Q_{i j}:=\frac{\left[\operatorname{Res}_{p}\left(\mathbb{V}^{(i, j)}(p, q) \psi_{j j}\right)\right] \psi_{i j}(q)-\left[\operatorname{Res}_{p}\left(\mathbb{V}^{(i, j)}(p, q) \psi_{i j}\right)\right] \psi_{j j}(q)}{\left[\operatorname{Res}_{p}\left(\mathbb{V}^{(i, j)}(p, q) \psi_{j i}\right)\right] \psi_{i j}(q)-\left[\operatorname{Res}_{p}\left(\mathbb{V}^{(i, j)}(p, q) \psi_{i i}\right)\right] \psi_{j j}(q)}, \quad i \neq j
$$

being the shifted rotation coefficients of the lattice. 
We can summarize the results of this section in

Theorem. The Miwa transformation generates a quadrilateral lattice with discrete rotation coefficients and renormalized tangent vectors given by

$$
\begin{aligned}
T_{j} Q_{i j} & =\frac{\left[\operatorname{Res}_{p}\left(\mathbb{V}^{(i, j)}(p, q) \psi_{j j}\right)\right] \psi_{i j}(q)-\left[\operatorname{Res}_{p}\left(\mathbb{V}^{(i, j)}(p, q) \psi_{i j}\right)\right] \psi_{j j}(q)}{\left[\operatorname{Res}_{p}\left(\mathbb{V}^{(i, j)}(p, q) \psi_{j i}\right)\right] \psi_{i j}(q)-\left[\operatorname{Res}_{p}\left(\mathbb{V}^{(i, j)}(p, q) \psi_{i i}\right)\right] \psi_{j j}(q)}, \quad i \neq j \\
\boldsymbol{e}^{(j)} & =\frac{1}{\operatorname{Res}_{p}\left(\mathbb{V}^{(j)}(p, q) \psi_{j j}\right)}\left(\mathbb{V}^{(j)}(p, q) \boldsymbol{X}_{j}-\frac{p}{q} \boldsymbol{X}_{j}\right) .
\end{aligned}
$$

\section{From orthogonal nets to circular lattices}

\subsection{Bilinear formulation of orthogonal nets}

The $N$-component BKP hierarchy can be formulated in terms of the following bilinear equation [8, 31]

$$
\int_{S^{1}} \psi(z, \boldsymbol{t}) \psi^{\mathrm{t}}\left(-z, \boldsymbol{t}^{\prime}\right) \frac{\mathrm{d} z}{2 \pi \mathrm{i} z}=G(\boldsymbol{t}) G\left(\boldsymbol{t}^{\prime}\right), \quad G(\boldsymbol{t})^{\mathrm{t}}=G(\boldsymbol{t})^{-1},
$$

where

$$
\boldsymbol{t}:=\left(\boldsymbol{t}_{1}, \ldots, \boldsymbol{t}_{N}\right), \boldsymbol{t}_{i}=\left(t_{i, 1}, t_{i, 3}, \ldots\right) .
$$

It is assumed that the Baker function $\psi$ admits a factorization

$$
\psi(z, \boldsymbol{t}):=\chi(z, \boldsymbol{t}) \psi_{0}(z, \boldsymbol{t})
$$

and that $\chi$ has a Laurent expansion on $S^{1}$ of the form

$$
\chi(z)=1+\beta z^{-1}+\mathcal{O}\left(z^{-2}\right)
$$

and

$$
\left.\psi_{0}(z, \boldsymbol{t})=\exp \left(\sum_{i=1}^{N} \xi\left(z, \boldsymbol{t}_{i}\right)\right) P_{i}\right), \quad \xi\left(z, \boldsymbol{t}_{i}\right)=\sum_{i=1}^{\infty} z^{2 n-1} t_{i, 2 n-1}
$$

As a consequence of (32) one finds that the rotation coefficients $\beta_{i j}$ and

the Baker function satisfy (6) and (8). Moreover one can identify $G(\boldsymbol{t})$ and 
$\left.\psi(z, \boldsymbol{t})\right|_{z=0}$ 31], thus $\left\{\boldsymbol{g}_{i}\right\}_{i=1}^{N}$ is an orthonormal frame and therefore the conjugate net is orthogonal; i. e., we have the Lamé equations for the rotation coefficients

$$
\begin{aligned}
& \frac{\partial \beta_{i j}}{\partial u_{k}}-\beta_{i k} \beta_{k j}=0, i, j, k=1, \ldots, N, \text { with } i, j, k \text { different } \\
& \frac{\partial \beta_{i j}}{\partial u_{i}}+\frac{\partial \beta_{j i}}{\partial u_{j}}+\sum_{\substack{k=1, \ldots, N \\
k \neq i, j}} \beta_{k i} \beta_{k j}=0, \quad i, j=1, \ldots, N, i \neq j
\end{aligned}
$$

There is again a vertex operator which plays a fundamental role in the analysis of geometrical transformations for the orthogonal net. It is given by

$$
\mathbb{V}^{(i)}(p) f(\boldsymbol{t}):=f\left(\boldsymbol{t}+[p] \boldsymbol{e}_{i}\right)
$$

where now

$$
[z]:=2\left(\frac{1}{z}, \frac{1}{3 z^{3}}, \ldots\right) .
$$

Indeed, as it is proved in [25], it follows that $\mathbb{V}^{(i)}(p)$ is related to the wellknown Ribaucour transformation in the form

$$
\mathcal{R}^{(i)}\left(\boldsymbol{g}_{j}\right):=(-1)^{\delta_{i j}} \mathbb{V}^{(i)}(p) \boldsymbol{g}_{j}=\boldsymbol{g}_{j}-\frac{2 \psi_{j i}(-p)}{\left|\mathfrak{C}^{(i)}\right|^{2}} \boldsymbol{C}^{(i)}
$$

where the Combescure vector is given by

$$
\boldsymbol{e}^{(i)}:=\sum_{k=1}^{N} \psi_{k i}(-p) \boldsymbol{g}_{k}
$$

\subsection{Miwa transformations and the circular lattice}

The circular lattice can be obtained from the the orthogonal net by discretizing variables in the Baker function through the Miwa transformation

$$
\Psi(z, \boldsymbol{t}, \boldsymbol{n}):=\psi(z, \boldsymbol{t}+\boldsymbol{n}[p])
$$

This property derives from the fact that (37) is a composition of Ribaucour transformations acting on tangent vectors of the orthogonal net, and 
therefore, as it was shown by Demoulin [10], it generates a circular lattice. The suitably renormalized tangent vectors are just the Combescure vectors $\left\{\mathbf{e}^{(i)}\right\}_{i=1}^{N}$. However, the computation of the coefficients $Q_{i j}$ of the corresponding circular lattice requires the use of the iteration : $\mathcal{R}^{(i, j)}=$ $\mathcal{R}^{(i)} \circ \mathcal{R}^{(j)}$. Thus we are lead to consider the composed vertex operator $\mathbb{V}^{(i, j)}(p)=\mathbb{V}^{(i)}(p) \circ \mathbb{V}^{(j)}(p)$ and to perform the substitutions $\boldsymbol{t} \rightarrow \boldsymbol{t}+[p]\left(\boldsymbol{e}_{i}+\boldsymbol{e}_{j}\right)$ and $\boldsymbol{t}^{\prime} \rightarrow \boldsymbol{t}$ in $(32)$.

Firstly, notice that

$$
\mathbb{V}^{(i, j)}(p) \psi(z)=\left[\mathbb{V}^{(i, j)}(p) \chi(z)\right] \psi_{0}(z)\left[1-\frac{2 z}{z-p}\left(P_{i}+P_{j}\right)\right] .
$$

This implies

$$
\operatorname{Res}_{p}\left(\mathbb{V}^{(i, j)}(p) \psi\right)=\operatorname{Res}_{p}\left(\mathbb{V}^{(i, j)}(p) \psi(z)\right)\left(P_{i}+P_{j}\right) .
$$

Moreover, the only non-vanishing contributions in (32) come from $z=\infty$ and $z=p$, as a consequence we have

$$
1-2\left(P_{i}+P_{j}\right)-\operatorname{Res}_{p}\left(\mathbb{V}^{(i, j)}(p) \psi\right) \psi^{\mathrm{t}}(-p)=\left[\mathbb{V}^{(i, j)}(p) G\right] G^{\mathrm{t}} .
$$

The orthogonal character of the right-hand side in the above formula gives

$$
\begin{gathered}
\left.\left(1-2\left(P_{i}+P_{j}\right)\right) \psi(-p)\right) \operatorname{Res}_{p}\left(\mathbb{V}^{(i, j)}(p) \psi^{\mathrm{t}}\right)+\operatorname{Res}_{p}\left(\mathbb{V}^{(i, j)}(p) \psi\right) \psi^{\mathrm{t}}(-p)\left(1-2\left(P_{i}+P_{j}\right)\right) \\
=\operatorname{Res}_{p}\left(\mathbb{V}^{(i, j)}(p) \psi\right) \psi^{\mathrm{t}} \psi \operatorname{Res}_{p}\left(\mathbb{V}^{(i, j)}(p) \psi^{\mathrm{t}}\right) .
\end{gathered}
$$

We use the same notation for the fundamental transformation as in the previous section, now:

$$
\Omega^{(i, j)}:=-\left[\operatorname{Res}_{p}\left(\mathbb{V}^{(i, j)}(p) \Phi\right)\right]^{-1} \Phi(-p) .
$$

This equation has two implications:

$$
\begin{gathered}
\Omega^{(i, j)}+\Omega^{(i, j)^{\mathrm{t}}}=\sum_{k=1}^{N} \tilde{\phi}_{k}^{\mathrm{t}}(-p) \tilde{\phi}_{k}(-p), \\
\tilde{\phi}_{k}(-p)=\operatorname{Res}_{p}\left(\mathbb{V}^{(i, j)}(p) \tilde{\phi}_{k}\right) \Omega^{(i, j)} .
\end{gathered}
$$

On the other hand, from (39) it follows

$$
\begin{aligned}
& \mathcal{R}^{(i, j)}\left(\boldsymbol{g}_{k}\right):=(-1)^{\delta_{i k}+\delta_{j k}} \mathbb{V}^{(i, j)}(p) \boldsymbol{g}_{k}=\boldsymbol{g}_{k}-(-1)^{\delta_{i k}+\delta_{j k}} \operatorname{Res}_{p}\left(\mathbb{V}^{(i, j)}(p) \tilde{\phi}_{k}\right)\left(\begin{array}{l}
\boldsymbol{C}^{(i)} \\
\boldsymbol{C}^{(j)}
\end{array}\right) \\
& =\boldsymbol{g}_{k}-\tilde{\phi}_{k}(-p) \Omega^{(i, j)^{-1}}\left(\begin{array}{l}
\mathbf{C}^{(i)} \\
\mathfrak{C}^{(j)}
\end{array}\right)
\end{aligned}
$$


as it should be. Observe that we have

$$
\begin{aligned}
\Omega_{i i}^{(i, j)} & =\frac{1}{2} \sum_{k=1}^{N} \psi_{k, i}^{2}(-p)=\frac{1}{2}\left|\mathfrak{C}^{(i)}\right|^{2}, \\
\Omega_{j j}^{(i, j)} & =\frac{1}{2} \sum_{k=1}^{N} \psi_{k, j}^{2}(-p)=\frac{1}{2}\left|\mathfrak{C}^{(j)}\right|^{2}, \\
\Omega_{i j}^{(i, j)}+\Omega_{j i}^{(i, j)} & =\sum_{k=1}^{N} \psi_{k, i}(-p) \psi_{k, j}(-p)=\mathbf{C}^{(i)} \cdot \mathbf{C}^{(j)} .
\end{aligned}
$$

Now, the Ribaucour transformation of the Combescure vector is

$$
\mathcal{R}^{(j)}\left(\mathfrak{C}^{(i)}\right)=\mathfrak{C}^{(i)}-2 \frac{\Omega_{i j}^{(i, j)}}{\left|\mathfrak{C}^{(j)}\right|^{2}} \mathfrak{e}^{(j)} .
$$

Hence, provided the discretization (37) is applied the transformation $\mathcal{R}^{(j)}=$ $T_{j}$ becomes the shift in the $n_{j}$ discrete variable and we get the quadrilateral lattice equations

$$
\Delta_{j} \mathfrak{e}^{(i)}=\left(T_{j} Q_{i j}\right) \mathfrak{e}^{(j)},
$$

where the rotation coefficients are given by:

$$
T_{j} Q_{i j}:=2 \frac{\operatorname{Res}_{p}\left(\mathbb{V}^{(i, j)}(p) \psi_{j j}\right) \psi_{i j}(-p)-\operatorname{Res}_{p}\left(\mathbb{V}^{(i, j)}(p) \psi_{i j}\right) \psi_{j j}(-p)}{\sum_{k=1}^{N} \psi_{k j}^{2}(-p)} .
$$

Moreover, the circularity property follows from the identity

$$
\mathfrak{C}^{(i)} \cdot \mathcal{R}^{(i)}\left(\mathfrak{C}^{(j)}\right)+\mathfrak{C}^{(j)} \cdot \mathcal{R}^{(j)}\left(\mathcal{C}^{(i)}\right)=2 \mathfrak{C}^{(i)} \cdot \mathfrak{e}^{(j)}-2\left(\Omega_{12}^{(i, j)}+\Omega_{12}^{(i, j)}\right)=0
$$

i.e,

$$
\mathfrak{C}^{(i)} \cdot T_{i}\left(\mathbf{C}^{(j)}\right)+\mathbf{C}^{(j)} \cdot T_{j}\left(\mathbf{C}^{(i)}\right)=0
$$

Summarizing:

Theorem. The Miwa transformation generates a circular lattice with discrete rotation coefficients and renormalized tangent vectors given by

$$
\begin{aligned}
T_{j} Q_{i j} & =2 \frac{\operatorname{Res}_{p}\left(\mathbb{V}^{(i, j)}(p) \psi_{j j}\right) \psi_{i j}(-p)-\operatorname{Res}_{p}\left(\mathbb{V}^{(i, j)}(p) \psi_{i j}\right) \psi_{j j}(-p)}{\sum_{k=1}^{N} \psi_{k j}^{2}(-p)} \\
\mathfrak{e}^{(j)} & =\sum_{k=1}^{N} \psi_{k j}(-p) \boldsymbol{g}_{k} .
\end{aligned}
$$




\section{References}

[1] L. Bianchi, Lezioni di Geometria Differenziale, 3-a ed., Zanichelli, Bologna (1924).

[2] A. Bobenko, in Symmetries and Integrability of Difference Equations II, P. Clarkson and F. Nijhoff (eds.), Cambridge University Press, Cambridge (1997).

[3] L. V. Bogdanov and B. G. Konopelchenko, J. Math. Phys. 39 (1998) 4701.

[4] J. Cieśliński, A. Doliwa and P. M. Santini, Phys. Lett. A235 (1997) 480.

[5] G. Darboux, Leçons sur la théorie générale des surfaces IV, GauthierVillars, Paris (1896). Reprinted by Chelsea Publishing Company, New York (1972).

[6] G. Darboux, Leçons sur les systèmes orthogonaux et les coordenées curvilignes (deuxième édition), Gauthier-Villars, Paris (1910) (the first edition was in 1897). Reprinted by Éditions Jacques Gabay, Sceaux (1993).

[7] E. Date, M. Jimbo, M. Kashiwara, and T. Miwa, J. Phys. Soc. Japan 50 (1981) 3806.

[8] E. Date, M. Jimbo, M. Kashiwara and T. Miwa, Physica D4 (1982) 343.

[9] E. Date, M. Jimbo and T. Miwa, J. Phys. Soc. Japan 51 (1982) 4116, 4125; 52 (1983) 388, 761, 766.

[10] M. A. Demoulin, Comp. Rend. Acad. Sci. Paris 150 (1910) 156.

[11] A. Doliwa, Quadratic reductions of quadrilateral lattices, solv-int/9802011.

[12] A. Doliwa, S. V. Manakov and P. M. Santini, $\bar{\partial}-$ Reductions of the Multidimensional Quadrilateral Lattice I: the Multidimensional Circular Lattice. Commun. Math. Phys. (in press). 
[13] A. Doliwa, M. Mañas, L. Martínez Alonso, E. Medina and P. M. Santini, Charged Free Fermions, Vertex Operators and Classical Theory of Conjugate Nets, solv-int/9803015.

[14] A. Doliwa and P. M. Santini, Phys. Lett. A 233 (1997) 365.

[15] A. Doliwa, P. M. Santini and M. Mañas, Transformations for Quadrilateral Lattices, solv-int/9712017.

[16] L. P. Eisenhart, A Treatise on the Differential Geometry of Curves and Surfaces, Ginn and Co., Boston (1909).

[17] L. P. Eisenhart, Trans. Amer. Math. Soc. 18 (1917) 111.

[18] L. P. Eisenhart, Transformations of Surfaces, Princeton University Press, Princeton (1923). Reprinted by Chelsea Publishing Company, New York (1962).

[19] E. I. Ganzha, On completeness of the Ribaucour transformations for triply orthogonal curvilinear coordinate systems in $\mathbb{R}^{3}$, solv-int/9606002.

[20] E. I. Ganzha and S. P. Tsarev, On superposition of the autoBäcklund transformations for (2+1)-dimensional integrable systems, solv-int/9606003.

[21] R. Hirota, J. Phys. Soc. Japan 50 (1981) 3785.

[22] H. Jonas, Berl. Math. Ges. Ber. Sitzungsber. 14 (1915) 96.

[23] V. G. Kac and J. van de Leur, in Important Developments in Soliton Theory, edited by A. S. Fokas and V. E. Zakharov (Springer, Berlin, 1993) p. 302.

[24] B. G. Konopelchenko and W. K. Schief, Three-dimensional Integrable Lattices in Euclidean Space: Conjugacy and Orthogonality, Preprint University of New South Wales (1997).

[25] G. Lamé, Leçons sur la théorie des coordenées curvilignes et leurs diverses applications, Mallet-Bachalier, Paris (1859).

[26] L. Lévy, J. l'École Polytecnique 56 (1886) 63. 
[27] Q. P. Liu and M. Mañas, Phys. Lett. A239 (1998) 159.

[28] Q. P. Liu and M. Mañas, J. Phys. A: Math. \& Gen. 31 (1998) L193.

[29] Q. P. Liu and M. Mañas, Superposition Formulae for the Discrete Ribaucour Transformations of Circular Lattices, Phys. Lett. A (in press).

[30] M. Mañas, A. Doliwa and P. M. Santini, Phys. Lett. A232 (1997) 365.

[31] M. Mañas and L. Martinez Alonso, From Ramond Fermions to Lamé Equations for Orthogonal Curvilinear Coordinates, Phys. Lett. B. (in press), solv-int/9805010.

[32] R. R. Martin, in The Mathematics of Surfaces, J. Gregory (ed.), Claredon Press, Oxford (1986).

[33] T. Miwa, Proc. Japan Acad. A58 (1982) 9.

[34] A. W. Nutborne, in The Mathematics of Surfaces, J. Gregory (ed.), Claredon Press, Oxford (1986).

[35] A. Ribaucour, Comp. Rend. Acad. Sci. Paris 74 (1872) 1489.

[36] M. Sato, RIMS Kokyuroku 439 (1981) 30; in Theta Functions-Bowdoin 198\%. Part 1 Proc. Sympos. Pure Maths. 49, part 1, 51, AMS (1989) Providence.

[37] R. Sauer, Differenzengeometrie, Springer-Verlag, Berlin (1970)

[38] G. Segal and G. Wilson, Publ. Math. I. H. E. S. 61, 5, 1985.

[39] V. E. Zakharov, On Integrability of the Equations Describing NOrthogonal Curvilinear Coordinate Systems and Hamiltonian Integrable Systems of Hydrodynamic Type I: Integration of the Lamé Equations Preprint (1996).

[40] V. E. Zakharov and S. V. Manakov, Func. Anal. Appl. 19 (1985) 11. 